\title{
Absorption kinetics and action profiles of mixtures of short- and intermediate-acting insulins
}

\author{
R. J.Heine ${ }^{1}$, H.J.G. Bilo ${ }^{1}$, T. Fonk ${ }^{1}$, E.A. van der Veen ${ }^{2}$ and J.van der Meer ${ }^{1}$ \\ Departments of ${ }^{1}$ Internal Medicine and ${ }^{2}$ Endocrinology, Free University Hospital, Amsterdam, The Netherlands
}

\begin{abstract}
Summary. The effects of mixing short- and intermediate-acting insulins (lente and NPH) on plasma insulin levels and action profiles, assessed by the euglycaemic clamp technique, were studied in 10 volunteers. Four protocols were used: (1) comparison between two semi-synthetic human soluble insulins in seven subjects $(0.22 \mathrm{IU} / \mathrm{kg})$; (2) assessment of insulin levels and action profiles of lente insulin in six subjects and of $\mathrm{NPH}$ insulin in five subjects $(0.33 \mathrm{IU} / \mathrm{kg})$; (3) comparison between mixtures of soluble with lente insulin and soluble with NPH insulin, administered immediately after mixing, in eight subjects $(0.55 \mathrm{IU} / \mathrm{kg}, 40 \%$ short-acting); (4) same mixtures, administered 2 days after preparation, in seven subjects. No differences in insulin levels and action profiles during the first $4 \mathrm{~h}$ after injection were found between both short-acting insulins and the soluble + NPH insulin mixtures. After the administration of NPH insulin, plasma insulin levels rose slightly
\end{abstract}

faster in comparison with lente insulin, with no significant differences between the action profiles for either insulin. Onset of action was delayed after soluble + lente insulin, both when administered immediately after mixing and to a greater extent when stored for 2 days before administration. After the latter procedure, the onset of action was markedly retarded and only slightly faster than after lente insulin alone.

We conclude, therefore, that mixing soluble with NPH insulin in a ratio of $2: 3$ does not affect the absorption kinetics of soluble insulin, whereas the onset of action is delayed when soluble is combined in the syringe with lente insulin, even when administered immediately after mixing.

Key words: Insulin therapy, soluble insulin, NPH insulin, lente insulin, human insulin, insulin miscibility, insulin absorption, insulin action, glucose clamp.
In clinical practice, patients with Type 1 (insulin-dependent) diabetes are often treated with a mixture of shortand intermediate-acting insulins.

NPH insulin is a neutral suspension of protamine insulin crystals [1]. Lente insulin is a mixture of preformed amorphous and crystalline insulins containing an excess of zinc [2]. The action profiles of both intermediate-acting insulins are very similar [3], but studies investigating the effect of mixing these insulins with soluble insulin on absorption kinetics have produced conflicting data $[4,5]$. In these studies in healthy volunteers, low insulin dosages were used in order to avoid hypoglycaemia. In addition, the large variability in insulin absorption rates made it difficult to investigate insulin miscibility in vivo.

The aim of this study was to compare, in normal volunteers, the effects on insulin profiles and insulin action of mixtures of soluble with NPH and soluble with lente insulins at dosages which are commonly used in the treatment of diabetes. To avoid counter-regulatory responses to hypoglycaemia and to assess insulin action, we used the manual euglycaemic clamp technique [6].

\section{Subjects and methods}

\section{Subjects}

We studied ten healthy male volunteers aged $20-30$ years (mean 23.3 years) and with a body mass index of $19.4-23.0 \mathrm{~kg} \div \mathrm{m}^{2}$ (mean $21.9 \mathrm{~kg} \div \mathrm{m}^{2}$ ). They were not suffering from any disease and had never received insulin. The study was approved by the local Ethical Committee and informed consent was obtained from all subjects. They were asked to take their usual diet and to fast $10 \mathrm{~h}$ before each study.

\section{Insulins}

The following insulin preparations were used: two semi-synthetic human soluble insulins (Velosulin Human, Nordisk, Gentofte, Denmark and Actrapid HM, Novo Industri, Bagsvaerd, Denmark), NPH insulin (Insulatard Human, Nordisk, Gentofte, Denmark) and lente insulin (Monotard HM, Novo, Bagsvaerd, Denmark). The protamine content of the NPH insulin was $0.13 \mathrm{mg} / \mathrm{ml}$ and the zinc content of the lente insulin $0.08 \mathrm{mg} / \mathrm{ml}$. For all studies, the same batches were used. The specific potencies of the insulins ranged from 38.4 to $40.0 \mathrm{IU} / \mathrm{ml}$, according to the specifications given by the manufacturers.

\section{Protocols}

Protocol 1. In seven subjects, the two soluble insulins were compared, using the same dosage as in protocols 3 and $4(0.22 \mathrm{IU} / \mathrm{kg}$ body weight).

Protocol 2. Plasma insulin levels and action profiles of lente insulin were assessed in six subjects and of NPH insulin in five of these subjects. The same dosage was used as in protocols 3 and $4(0.33 \mathrm{IU} / \mathrm{kg}$ body weight).

Protocol 3. In eight subjects, mixtures of soluble (Actrapid HM) with lente insulin and soluble (Velosulin Human) with NPH insulin were compared. The total administered insulin dosage was $0.55 \mathrm{IU} / \mathrm{kg}$ body weight, of which $40 \%$ was short-acting insulin. The insulins were administered immediately (within $1 \mathrm{~min}$ ) after preparing the mixtures.

Protocol 4. In seven subjects, the stability of the same mixtures was studied. The mixtures were stored in the syringe for $48 \mathrm{~h}$ at $4^{\circ} \mathrm{C}$ before injection.

For each subject the tests were separated by at least one week and the order of the tests was randomised for protocols 1, 3 and 4 . 


\section{Study design}

On the morning of each study, two intravenous cannulae (Venflon, Viggo, Helsingborg, Sweden) were inserted into the antecubital fossa of each arm. One was used for intermittent sampling of blood glucose and hormone levels and was kept patent with saline $(0.15 \mathrm{~mol} / 1)$. The second cannula was used for the infusion of glucose $(1.11 \mathrm{~mol} / \mathrm{l})$ by a variable rate volumetric infusion pump (Imed 928, Abingdon, Oxon, UK). At time zero, insulin was injected subcutaneously and perpendicularly in the front of the thigh using a $1 \mathrm{ml}$ Plastipak syringe (Becton Dickinson, Run Laoghaire, Dublin, Ireland). Throughout the experiments, the subjects remained in a supine position and abstained from food, drink and smoking.

After the insulin injection, blood glucose values were maintained at a fasting blood glucose level $(0.5 \mathrm{mmol} / \mathrm{l})$ by performing a 'manual' glucose clamp, to prevent endogenous insulin secretion $[6,7]$. Blood glucose values were fed into a preprogrammed calculator (Model T I 59, Texas Instruments, Dallas, USA) every $5 \mathrm{~min}$. As soon as blood glucose levels had declined by $0.3 \mathrm{mmol} / 1$ below the fasting level, the glucose infusion was initiated at a rate of $0.005 \mathrm{mmol} \cdot \mathrm{kg}^{-1} \cdot \mathrm{min}^{-1}$. Thereafter, glucose infusion rates were adjusted according to the equation derived from that originally described by DeFronzo et al. [7] with supplementary infusion when blood glucose levels decreased by $>0.2 \mathrm{mmol} / 1$ in $5 \mathrm{~min}[6]$. The glucose clamps were maintained for $8 \mathrm{~h}$ after the administration of the insulin mixtures and intermediateacting insulins, and for $4 \mathrm{~h}$ after the injection of the soluble insulins.

\section{Methods}

Venous blood samples were taken for blood glucose, plasma insulin and C-peptide levels at indicated intervals. Blood glucose was measured by a glucose oxidase method (Yellow Springs Instruments, Yellow Springs, Ohio, USA). Plasma insulin was assessed by radioimmunoassay with human insulin as standard (Sorin Biomedica, Saluggia, Italy; sensitivity $2.5 \mathrm{mU} / 1$; intra-assay coefficient of variation $8.2 \%$ ). Plasma C-peptide was measured by radioimmunoassay with ethanol precipitation with human C-peptide as standard (Novo Industri, Bagsvaerd, Denmark; sensitivity $0.06 \mathrm{nmol} / 1$, intra-assay coefficient of variation $8.1 \%$ ) [8].

\section{Calculations}

Results are expressed as mean $\pm \mathrm{SEM}$, or as indicated. For the statistical evaluations, the Student's t-test for paired and unpaired observations was used for the comparisons within and between protocols, respectively. A quantitative estimate of insulin action was provided in the euglycaemic clamp by the glucose requirements, expressed as $\mathrm{mmol} \cdot \mathrm{kg}^{-1} \cdot \mathrm{min}^{-1}$. Glucose infusion rates were calculated for 15-min intervals. Areas under the glucose infusion rate curves were calculated with the formula:

area $=\frac{\Sigma\left(\mathrm{X}_{\mathrm{n}+1}-\mathrm{X}_{\mathrm{n}}\right)\left(\mathrm{Y}_{\mathrm{n}}+\mathrm{Y}_{\mathrm{n}+1}\right)}{2}$

where $X_{n}=$ sampling time in minutes at time point $n$ and $Y_{n}=$ glucose infusion rate during $5 \mathrm{~min}$ from time point $\mathrm{n}$.

\section{Results}

The mean deviation from the intended clamp value calculated for all glucose clamps was $0.05 \pm 0.05 \mathrm{mmol} / 1$ (mean $\pm S D$ ) and the mean coefficient of variation of the blood glucose values was $5.7 \pm 1.5 \%$ (mean $\pm S D$ ). Plasma C-peptide levels remained below the fasting concentrations throughout the clamps, excluding the occurrence of stimulated endogenous insulin secretion (Fig. 1).
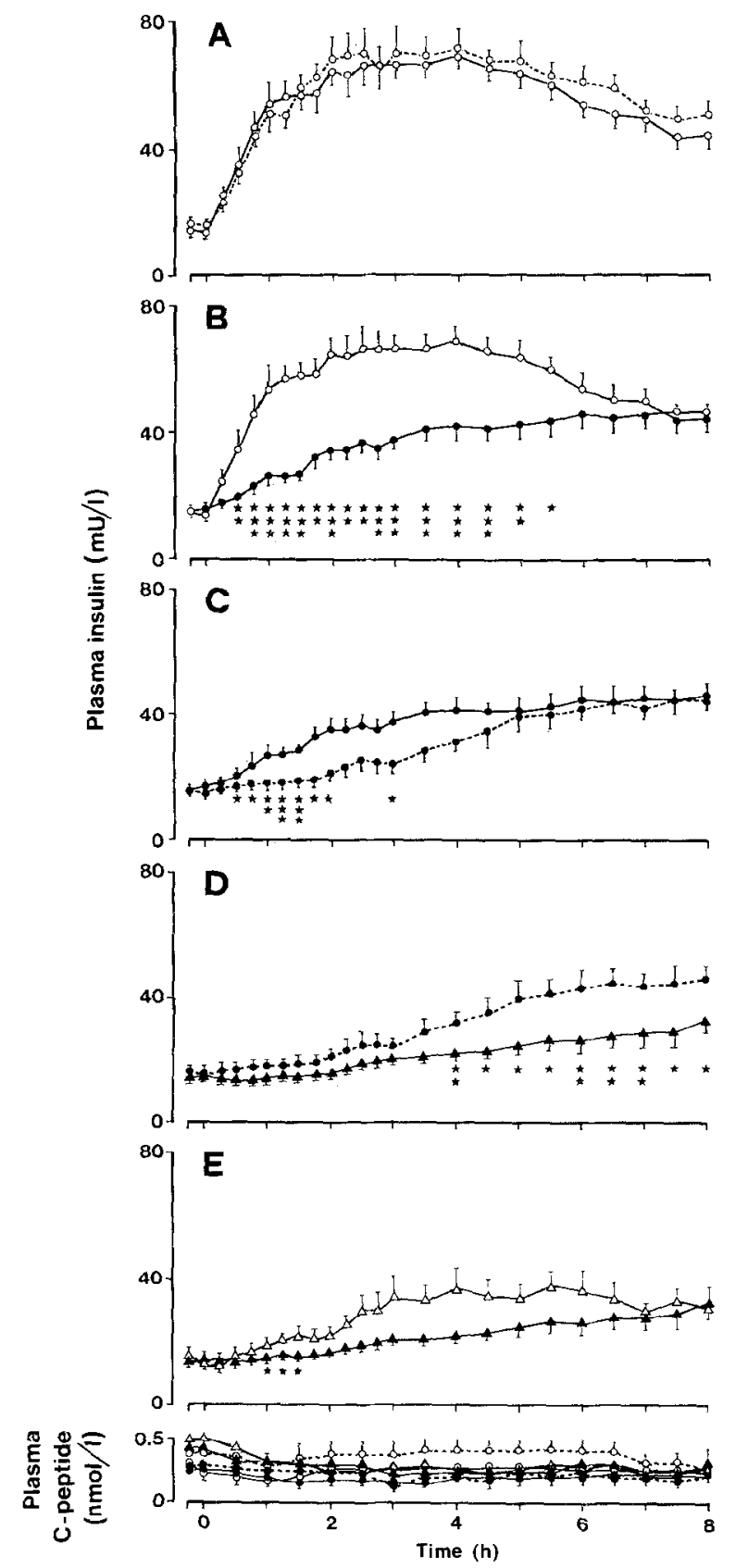

Fig. 1.A-E. Plasma insulin and C-peptide levels during glucose clamping after the administration of the insulin mixtures and intermediate-acting insulins alone. A soluble $+\mathrm{NPH}$ insulin, immediately $(\mathrm{O}-\mathrm{O})$ and 2 days $\left(\mathrm{O}-\mathrm{O}_{-}\right)$after mixing; $\mathrm{B}$ soluble $+\mathrm{NPH}$ insulin $(\mathrm{O}-\mathrm{O})$ and soluble + lente insulin $(-\infty)$, immediately after mixing; $\boldsymbol{C}$ soluble + lente insulin, immediately $(-\infty)$ ) and 2 days ( - - ) after mixing; $\mathbf{D}$ lente insulin alone $(\boldsymbol{\Lambda})$ and soluble + lente insulin, 2 days after mixing (-.--); E lente insulin $(\Delta-\Delta)$ and NPH insulin $(\Delta-\Delta)$ alone. ${ }^{*} p<$ $0.05 ; * * p<0.02 ; * * *<<0.01$

\section{Comparison between the effects on plasma insulin levels and action profiles of the short- and intermediate-acting insulins}

Plasma insulin levels and glucose requirement during the glucose clamps were not significantly different at any time point for either soluble insulin. The adminis- 

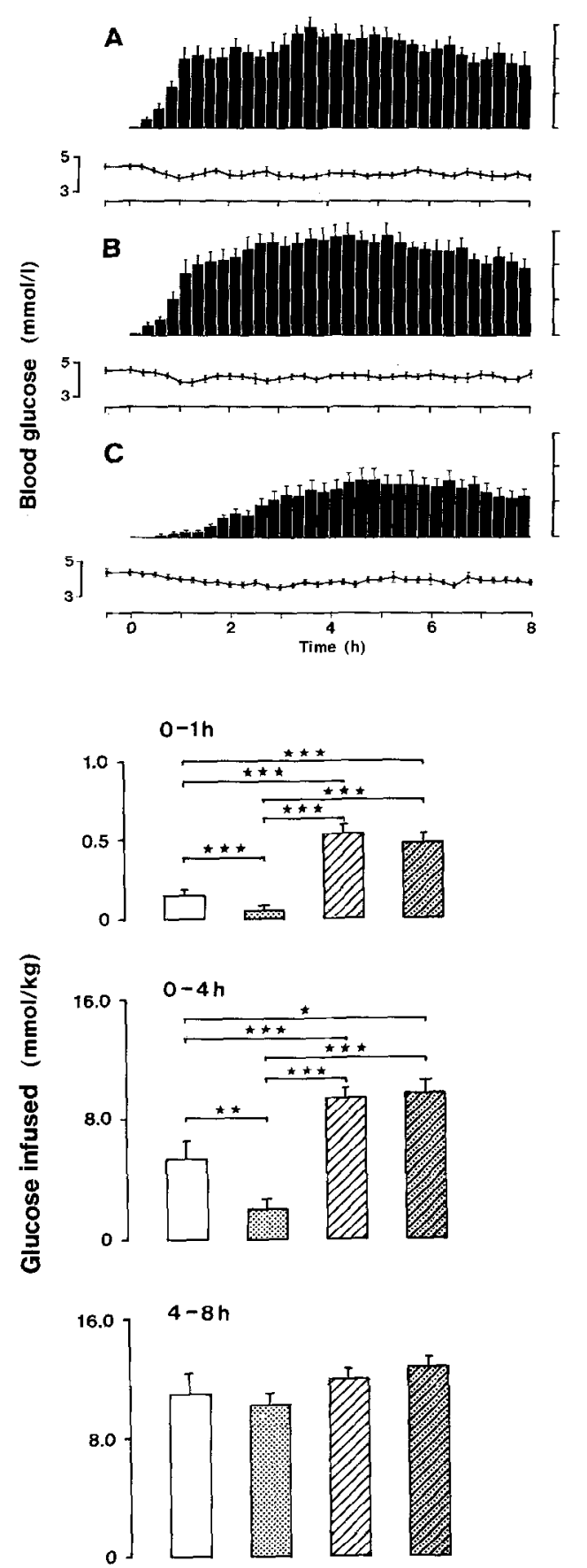

Fig.3. Areas under the glucose infusion rate curves, calculated for the intervals $0-1 \mathrm{~h}, 0-4 \mathrm{~h}$ and $4-8 \mathrm{~h}$, after the administration of soluble + lente immediately after mixing $(\square)$; soluble + lente 2 days after mixing ( : $)$; soluble + NPH insulin immediately after mixing $(\square)$ ); soluble + NPH insulin 2 days after mixing ( $\left.\mathbb{R}^{*}\right)$. ${ }^{*} p<0.05 ;{ }^{* *} p<0.02$; $* * * p<0.01$

tration of NPH insulin was followed by a slightly faster rise of the plasma insulin levels compared with lente insulin ( $15 \pm 2$ to $19 \pm 2$ and $15 \pm 2$ to $15 \pm 1 \mathrm{mU} / 1$ at $60 \mathrm{~min}$, respectively; $p<0.05$; Fig. $1 \mathrm{E}$ ). Plasma insulin levels were significantly higher after NPH insulin compared with lente insulin $(60-90 \mathrm{~min}$; all $p<0.05)$. Glucose requirements during the glucose clamps were not significantly different for any 15 -min interval for either intermediate-acting insulins (Fig. 2).

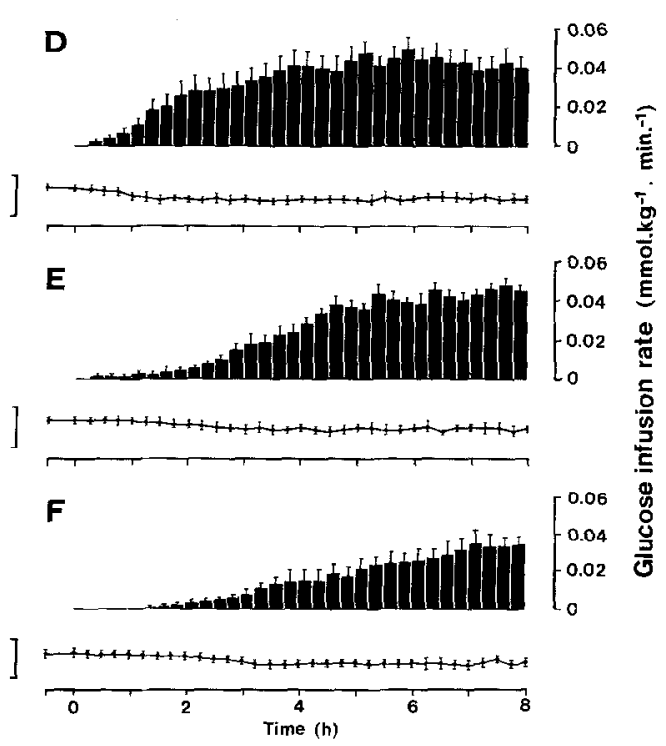

Fig. 2A-F. Blood glucose levels and glucose infusion rates during the glucose clamps after the administration of $\mathbf{A}$ soluble + NPH insulin immediately after mixing; B soluble + NPH insulin 2 days after mixing; C NPH insulin alone; $\mathbf{D}$ soluble + lente insulin immediately after mixing; E soluble + lente insulin 2 days after mixing; F lente insulin alone
Comparison between the effects on plasma insulin levels and action profiles of the insulin mixtures

Plasma insulin levels for soluble + lente insulin and soluble + NPH insulin studies are shown in Figure 1. After the administration of soluble + NPH insulin, compared with soluble + lente insulin, plasma insulin levels increased faster during the first hour $(15 \pm 1$ to $55 \pm 7$ and $16 \pm 1$ to $27 \pm 3 \mathrm{mU} / 1$, respectively; $p<0.01$ ).

Two hours after the administration of soluble + NPH insulin, a plateau was reached of approximately $67 \mathrm{mU} / 1$ for another $2 \mathrm{~h}$, after which plasma insulin levels decreased to $45 \pm 5 \mathrm{mU} / 1$ at $8 \mathrm{~h}$. After the administration of soluble + lente insulin, plasma insulin levels increased gradually during $6 \mathrm{~h}$ to a plateau level of approximately $46 \mathrm{mU} / 1$. Plasma insulin levels after soluble + lente insulin, compared with soluble + NPH insulin, were significantly lower from 30 to $330 \mathrm{~min}$ (Fig. 1 B).

Glucose requirements and blood glucose levels during the 8-h glucose clamps for the soluble + lente insulin and soluble + NPH insulin studies are shown in Figure 2. The action profiles of both studies resemble the corresponding plasma insulin curves. The areas under the curves, calculated for the intervals $0-1$ and $0-4 \mathrm{~h}$, were significantly greater after the immediate administration of soluble + NPH insulin in comparison with soluble + lente insulin $[0.594 \pm 0.078$ versus $0.161 \pm$ $0.028 \mathrm{mmol} / \mathrm{kg} \quad(p<0.01)$ and $9.722 \pm 0.661$ versus $5.661 \pm 1.044 \mathrm{mmol} / \mathrm{kg}(p<0.01)$, respectively; Fig.3]. The areas under the curve from 4 to $8 \mathrm{~h}$ were not significantly different in either study.

Plasma insulin levels were not significantly different at any time point after the administration of soluble + NPH insulin combinations, immediately and 2 days after mixing (Fig. 1A). The administration of soluble + lente insulin, 2 days after mixing, resulted in a delay in the plasma insulin rise by approximately $2 \mathrm{~h}$, compared with injection immediately after mixing. Plasma insulin levels were significantly lower at all time points between 30 and $120 \mathrm{~min}$ and at $180 \mathrm{~min}$ when administered at 

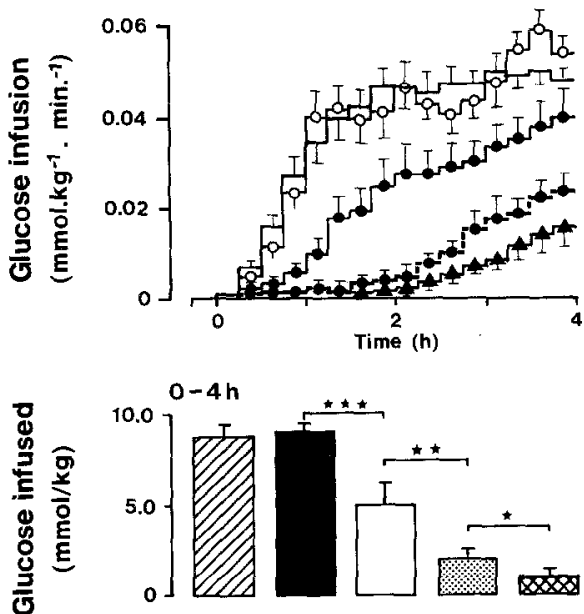

Fig.4. Glucose infusion rates and areas under the glucose infusion rate curves from 0 to $4 \mathrm{~h}$ after the administration of soluble $+\mathrm{NPH}$ insulin immediately after mixing $(\mathrm{O}-\mathrm{O}, \square)$; soluble alone $(-\square)$; soluble + lente immediately after mixing $(\bullet-\longrightarrow, \square)$;

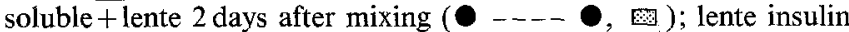
alone $(\boldsymbol{\Lambda}, \mathbf{\Lambda}) .{ }^{*} p<0.05 ;{ }^{* *} p<0.02 ;{ }^{* *} p<0.01$

2 days, compared with immediately after mixing (Fig. 1C). The insulin plateaux were similar for both soluble + lente insulin studies from 6 to $8 \mathrm{~h}$, suggesting a shift to the right of the plasma insulin curve by $2 \mathrm{~h}$ after the administration of soluble + lente insulin 2 days after mixing. Plasma insulin levels were significantly lower from 240 to $480 \mathrm{~min}$ after lente insulin alone, compared with soluble + lente insulin, administered 2 days after mixing (Fig. 1D).

The glucose requirement profiles were very similar for both soluble + NPH insulin studies (Fig. 2). In addition the areas under the curves for the first hour, from 0 to $4 \mathrm{~h}$, and from 4 to $8 \mathrm{~h}$, were not significantly different for either study (Fig. 3). After soluble + lente insulin, injected 2 days after mixing, the glucose requirements were significantly less than after the immediate administration, in the first hour and from 0 to $4 \mathrm{~h}[0.039 \pm$ 0.028 versus $0.161 \pm 0.028 \mathrm{mmol} / \mathrm{kg} \quad(p<0.01)$ and $2.178 \pm 0.389$ versus $5.661 \pm 1.044 \mathrm{mmol} / \mathrm{kg}(p<0.02)$, respectively]. The areas under the curves from 4 to $8 \mathrm{~h}$ were not significantly different either for the soluble + lente insulin study.

Glucose requirements $(0-4 \mathrm{~h})$ were not significantly different after the administration of soluble alone or soluble + NPH insulin, whereas a significant decline of the glucose requirements was seen in the following order: soluble alone; soluble + lente insulin administered immediately $(p<0.01)$; soluble + lente insulin injected 2 days after mixing $(p<0.02)$; and lente insulin alone $(p<0.05$; Fig. 4$)$.

\section{Discussion}

This study demonstrates that lente insulin delays the onset of action of soluble insulin, both when mixed immediately before injection, and to a greater extent when the combination is stored for 2 days before administra- tion. Mixtures of soluble and NPH insulins were stable for 2 days and no delay in the onset of action of soluble insulin was observed when compared with the administration of soluble alone. The latter results are in agreement with a previously reported study in which the disappearance rate of 125 -iodine labelled soluble insulin was not altered when injected subcutaneously in combination with NPH insulin [9]. However, in studies using this indirect methodology, it has to be assumed that iodinated insulin behaves like unlabelled insulin and that the rate of disappearance of externally counted radioactivity is proportional to the amount of absorbed insulin. Furthermore, as degradation of insulin at the injection site can influence the assessment of insulin absorption, one has to assume that no degradation takes place. The validity of these assumptions are still considered to be controversial [4].

In order to assess the effect of mixing insulins on the absorption kinetics, it is either necessary to determine the plasma insulin levels and insulin action profiles of the used insulins separately, or to inject the soluble and intermediate-acting insulins into contralateral thighs. The advantage of the former method is the possibility to compare the profiles of the single insulins.

Since the effects of the administration of both soluble insulins (Actrapid HM and Velosulin Human) on plasma insulin levels and action profiles were similar, this excludes a possible explanation for the observed differences. The absorption of NPH insulin was slightly faster than that of lente insulin; however no significant differences were found in the action profiles. It is evident that this small difference, in the absorption rate between intermediate-acting insulins, cannot explain the markedly retarded onset of action of the soluble + lente insulin mixtures. As shown in Figures 2 and 4, the insulin action during the first $4 \mathrm{~h}$ after the administration of the insulin mixtures is determined mostly by the shortacting insulins.

The great differences in glucose requirements during the glucose clamps following the soluble + lente insulin and soluble + NPH insulin administrations can be explained by the steep insulin dose response curves in the physiological range of plasma insulin levels, where small differences in insulin concentrations are translated into large alterations in glucose uptake [10].

Our results suggest that soluble insulin, when mixed with lente, is bound by the excess of zinc, and that this binding increases with time. After storing the mixture for 2 days the pharmacokinetic properties of soluble insulin are almost completely altered, as demonstrated by the comparison of the plasma insulin levels and action profiles after the administrations of the soluble + lente insulin mixture 2 days after mixing, and lente insulin alone. Our results are supported by the in vitro findings of Grootendorst et al., who demonstrated an $80 \%$ binding of the soluble insulin by the excess of zinc in 3 min after mixing soluble with zinc insulin in the proportions $1: 3$ and $1: 2$ [11]. In contrast to our observations, Galloway et al. demonstrated in a combined in vivo and in vitro study that changing the soluble: NPH 
insulin ratio from $1: 3$ to $1: 1$ resulted in a gradual increase of plasma insulin levels and hypoglycaemic effect, whereas in vitro a considerable proportion of the added soluble insulin was bound to NPH insulin. The same experiments with lente and soluble insulin showed, when the proportion of soluble was increased, a minimal binding of soluble insulin to lente, while the enhancement of the clinical effects was negligible [5]. These in vitro results do not agree with the findings of Grootendorst et al., possibly due to different methods used in these studies [5,11]. Binding of soluble insulin to NPH insulin is probably also influenced by the formulation, especially in terms of protamine content and other additives used [12]. These factors may explain partly the differences in results in comparison to those of Galloway et al. Since binding of soluble insulin in vitro to zinc or NPH is not necessarily stable, it is not possible to predict in vivo absorption kinetics from these results. This may also explain the discrepancy between the in vivo and in vitro results of Galloway et al. [5].

Berger et al. [4] concluded from a study in six normal volunteers that lente insulin does not influence insulin absorption kinetics of soluble insulin when administered directly after mixing, but if injected $5 \mathrm{~min}$ later a slower rise of the insulin levels was observed. As in our study, no effect was seen when combining NPH with soluble insulin on the insulin profiles. The insulin preparations were similar to the ones we used, but the dosages were lower, i.e. $10 \mathrm{U}$ soluble and $16 \mathrm{U}$ intermediate-acting insulin. The higher dosage in our study might have influenced the absorption kinetics. A greater volume may disperse slower, because of the smaller surface to volume ratio, which possibly enhances the interaction between the excess of zinc and soluble insulin. The different results of our study compared with those of Berger et al. are not explained by the fact that we used human insulin, because the zinc content of porcine and human lente insulin is identical. Further studies are necessary to establish the effect of other dosages, varying proportions of soluble and intermediate-acting insulin, and different formulations of NPH and lente insulin on absorption kinetics.

In summary, mixtures of soluble with NPH insulin can be stored for at least $48 \mathrm{~h}$ without influencing the absorption kinetics of soluble insulin. Lente insulin delays the onset of action of soluble insulin when mixed in one syringe, both when prepared immediately before and to a far greater extent when stored for 2 days prior to administration.

We conclude therefore that mixing soluble with NPH insulin in a ratio of $2: 3$ does not affect the absorption kinetics of soluble insulin, whereas the onset of ac- tion is delayed when soluble is combined with lente insulin, even when administered immediately after mixing.

Acknowledgements. We are grateful to Mrs. M.Z.Lomecky and Mr. C.P. de Vries for the insulin and C-peptide assays and to Mrs. L.J. Koster for preparing the manuscript.

\section{References}

1. Krayenbïhl CH, Rosenberg Th (1946) Crystalline protamine insulin. Rep Steno Hosp (Kbh) I: 60-73

2. Hallas-Møller K, Petersen K, Schlichtkrull J (1951) Crystalline and amorphous insulin zinc compounds with prolonged action. Ugeskr Laeg 113: 1761-1767

3. Poulsen JE, Deckert T (1976) Insulin preparations and the clinical use of insulin. In: Luft R (ed) Insulin. Islet pathology, islet function and insulin treatment. A lindgren \& Söner, Mölndal, Sweden, pp 197-245

4. Berger M, Cüppers HJ, Hegner H, Jörgens V, Berchtold P (1982) Absorption kinetics and biologic effects of subcutaneously injected insulin preparations. Diabetes Care 5: 77-91

5. Galloway JA, Spradlin CT, Jackson RL, Otto DC, Bechtel LD (1982) Mixtures of intermediate acting insulin (NPH and lente) with regular insulin: an update. In: Skyler JS (ed) Insulin update: 1982, Excerpta Medica, Amsterdam, pp 111-120

6. Ponchner M, Heine RJ, Pernet A, Hanning I, Francis AJ, Cook D, Ørskov H, Alberti KGMM (1984) A comparison of the artificial pancreas (glucose controlled insulin infusion system) and a manual technique for assessing insulin sensitivity during euglycaemic clamping. Diabetologia 26:420-425

7. DeFronzo RA, Tobin JD, Andres R (1979) Glucose clamp technique: a method for quantifying insulin secretion and resistance. Am J Physiol 237: E 214-223

8. Heding $\mathcal{L}$ (1975) Radioimmunological determination of human $C$ peptide in serum. Diabetologia 11: 541-548

9. Kolendorf K, Bojsen J, Deckert T (1983) Absorption and miscibility of regular porcine insulin after subcutaneous injection of insulin treated diabetic patients. Diabetes Care 6:6-9

10. Rizza R, Mandarino L, Gerich J (1981) Dose-response characteristics for the effects of insulin on production and utilization of glucose in man. Am J Physiol 249: 630-639

11. Grootendorst BC, Jonkers JR, Kruit I (1983) The miscibility of short acting insulins with depot-insulins. An in vitro study. Pharmaceutisch Weekblad 118: 746-751

12. Nolte MS, Poon V, Grodsky GM, Forsham PH, Karam JH (1983) Reduced solubility of short-acting soluble insulins when mixed with longer-acting insulins. Diabetes 32: 1177-1181

Received: 17 February 1984

and in revised form: 15 September 1984

Dr. R. J. Heine

Department of Internal Medicine

L 236 Meander

Free University Hospital

De Boelelaan 1117

P.O. Box 7057

NL-1007 MB Amsterdam

The Netherlands 